\title{
Study of medium range reordering by plastic deformation in $\mathrm{Cu}_{46} \mathrm{Zr}_{46} \mathrm{Al}_{8}$
}

J. Enrique Velasco ${ }^{\mathrm{a}}$, Amadeu Concustell $^{\mathrm{b}}$, Eloi Pineda ${ }^{\mathrm{a}}$, Daniel Crespo ${ }^{\mathrm{a}}$

${ }^{a}$ Dept. Física, Universitat Politècnica Catalunya - BarcelonaTech, 08860 Castelldefels, Catalonia, Spain.

je.velasco85@gmail.com / eloi.pineda@upc.edu / daniel.crespo@upc.edu

${ }^{\mathrm{b}}$ Eurecat, Plaça de la Ciència 2, 08243 Manresa, Spain

aconcustell@hotmail.com

*Corresponding author:

J. Enrique Velasco

je.velasco85@gmail.com

Tel. (34) 934134141

\begin{abstract}
The influence of shear in the atomic structure of ternary $\mathrm{Cu}_{46} \mathrm{Zr}_{46} \mathrm{Al}_{8}$ metallic glass was studied at different temperatures by molecular dynamics (MD) simulation. At temperatures above and below the glass transition temperature the system was subjected to a shear deformation cycle; the shear deformation was carried in the [100] direction and then the original geometry was recovered. The system was analyzed in three states: initial state (before deformation), deformed state (sheared) and final state (recovery). The different states obtained by the atomistic simulations were examined by computing the directional pair distribution functions (dPDF) in the coordinate planes. The results showed by dPDFs of the planes perpendicular to the coordinate axis are apparently isotropic. However, the dPDF disengage when computed perpendicular to rotated axis. This reveals that the anisotropy introduced during the deformation cycles appears in directions [110] and [1 $\overline{1} 0]$, tilted $45^{\circ}$ with respect to the direction of the applied shear. To analyze the subtle structural change we systematically computed the positions and widths of the dPDF peaks on the rotated directions, thence allowing us to describe in detail the different signatures of anisotropy induced by deformation in the metallic glasses.
\end{abstract}


Keywords: Amorphous alloys, Aging and rejuvenation, Anisotropy, Mechanical deformation

\section{Introduction}

Intense efforts have been made to understand metallic glasses (MGs); their formation, deformation process, fracture behavior and other aspects of their nature and properties are currently the focus of intense research. The amorphous structures of MGs are characterized by the random, disordered atomic arrangement, lacking the principal directions of crystal lattices. However, since the very beginning of metallic glass research the anisotropy of their properties and structure was noticed; the extreme high cooling rates and non-isotropic quenching conditions of the most common production techniques imply, in fact, the presence of structural anisotropy [1]. In addition, posterior thermal and mechanical treatments applied with the aim of modifying some glass properties [2] may erase or promote anisotropy. MGs have high elastic strain, high strength and high fracture toughness that make them useful in structural and functional applications [3][4][5][6]. Special interest is focused on the use of these materials in small parts and micro-electromechanical systems; their lack of microstructure combined with outstanding elastic and mechanical properties down at the microscale [7] open exciting new opportunities of design. The presence of elastic anisotropy [8], induced either by the production method or by posterior thermo-deformation [9], is therefore of major interest in order to control the mechanical properties of these devices as well as to open new possibilities of fine, directional tuning of the mechanical response. Furthermore, maybe the most salient feature of glasses is that very subtle, almost imperceptible, changes in the structure lead to enormous effects on their dynamics. The most striking example is the glass transition, in which small changes of the liquid structure are accompanied by changes of several order of magnitudes in viscosity. Also in the glassy state; small structural changes driven by physical aging may change the glass relaxation dynamics [10] and affect some important properties like transforming the fracture behavior from ductile to brittle [11]. In this 
framework, it is expected that a small anisotropy in the glass structure could induce perceptible anisotropy of some macroscopic properties.

The experimental access to glass structure is obtained through scattering techniques, giving us direct information on the reciprocal space. In most cases the obtained experimental information is averaged over atomic species as well as over several geometrical directions. Therefore, it is a complex task to experimentally reveal and typify the anisotropic features in a glass structure [12]. The elastic affine deformations during insitu tensile or compression experiments, with the associated anisotropy between the directions along and across the loading axis, have been well characterized by diffraction experiments [13]. On the other hand, the permanent remnant anisotropy of the unloaded samples after a plastic deformation or creep experiment are more subtle and difficult to identify [14][15]. Molecular dynamics help us to study in depth the atomic scale structure of glasses with a detail not available experimentally. The short range order (SRO) rearrangements during deformation, elastic and plastic, have been extensively studied by atomistic simulation. Compact configurations such as icosahedral atomic coordination have been connected to more rigid clusters, i.e. local zones less prone to comply under external stress, generating a heterogeneous scenario in which deformation localizes in softer environments [16]. The study of structural indicators that define and allow us to characterize the diverse short range order configurations is important and has received much attention. The statistical distribution of such local configurations, and its evolution during quenching, annealing/aging and deformation reveals the trends in SRO associated to the changes of the macroscopic properties [17][18]. An important, still on-going task is to relate the short order analysis coming from simulations to the observable changes in the structure factor $S(q)$ or the pair distribution function $g(r)$ obtained from experiments.

In this work we analyze molecular dynamics simulations of a $\mathrm{Cu}_{46} \mathrm{Zr}_{46} \mathrm{Al}_{8}$ metallic glass to investigate the spatial atomic distribution under the effect of shear deformation cycles at different isotherms. In a previous study we simulated the process in a Pd-based metallic glass [19], following the same procedure here we computed the partial pair correlation functions on parallel planes with different orientations relative to a shearing axis. As in the Pd-based case, the main differences were observed in the second and third neighboring shell. The results in the previous work showed remnant anisotropy in several 
cases, but not defined trends were observed. In this work we systematically analyze the different deformation cycles, temperatures and the obtained directional pair distribution functions (dPDF) characterizing the signatures of the remnant anisotropy in the short and medium range order.

\section{Simulations details}

MD simulations were performed by using the open source LAMMPS numerical code developed at Sandia National Laboratories [20]. The potential used was the embedded atom method (EAM) developed for amorphous $\mathrm{ZrCuAl}$ alloys in ref. [21]. The $\mathrm{Cu}_{46} \mathrm{Zr}_{46} \mathrm{Al}_{8}$ composition was chosen because it has been shown to be a good glass-former alloy [22] and also because of the $\mathrm{Cu}-\mathrm{Zr}-\mathrm{Al}$ system has been extensively studied by means of MD simulations, thus giving us good confidence in the validity of the EAM potential parameters [21][23][24]. The sample, with aspect ratio $5 \times 5 \times 1$ and containing $N_{a}=2 \times 10^{5}$ atoms, was first melted at $\mathrm{T}=2000 \mathrm{~K}$ and equilibrated for $2 \mathrm{~ns}$. The glassy state was prepared by quenching at a rate of $10^{12} \mathrm{~K} \mathrm{~s}^{-1}$. After the quenching process, samples were hold at the final temperature for 2 ns. Final temperatures of 800, 740, 720, 700, 680, 650, 500, 450, 400 and $300 \mathrm{~K}$ were used to perform the deformation cycles at different isotherms. The equilibrated samples were subjected to a shear deformation cycle with the shear direction parallel to the $x$ axis ([100] direction), at a deformation rate of $\dot{\gamma}=10^{10} \mathrm{~s}^{-1}$. Periodic boundary conditions were used trough the simulation process. The simulation time step was $0.001 \mathrm{ps}$. The deformation cycles were performed in NPT conditions, with maximum shear deformation of $\varepsilon_{x}=d x / L x=10,25$ and $40 \%$ respective to the box length (see Fig. 1). Shear was applied by holding fix the lower surface and displacing the upper one in the shear direction; the corresponding affine displacement of each atom is added to that due to the thermal movement.

The atomic directional pair distribution function (dPDF) is computed in planes or slices perpendicular to defined axes. The dPDF is defined as

$$
g(r)=\frac{1}{N_{a}} \sum_{i}^{N_{a}} \frac{1}{N_{b}} \frac{1}{2 \pi r \Delta} \sum_{j}^{N_{b, i}}\left(\left\langle\delta\left(\left|\vec{r}_{i j}\right|-r\right)\right\rangle\right)
$$


Here, $N_{b, i}$ is the number of atoms in a plane section of thickness $\Delta$ to which the atom $i$ belongs to, and $\vec{r}_{i j}$ is the interatomic distance between the $i j$-atomic pair in the same box slice. The thickness of the slices was fixed as $\Delta=2 \AA$, the distance in which the PDF is null. Thence, we will use the following notation, [hkl]-dPDF is the PDF in the planes normal to the [hkl] direction. In order to reveal changes in the dPDF after different deformation and detect medium range order changes, we systematically computed the peak positions $r_{i}$ and widths $\Gamma_{i}$ of each $i$ th peak of the dPDFs. The whole systematic analysis was performed on dPDFs perpendicular to normal $x, y$ and $z$ axes and in axes belonging to the $x y$ plane and rotated $22.5^{\circ}, 45^{\circ}$ and $67.5^{\circ}$ around the z-axis.

\section{Results and discussion}

Figure 1 shows an example of the initial box and a shear deformed box at $\varepsilon_{x}=25 \%$, the definition of the [100], [010] and [001] directions is also specified there. Here we recall that the aim of this work is to analyze the remnant effects of the complete deformation cycle (initial - sheared - recovered) that includes the recovery process at the same shearing rate. Fig. 1 shows the PDFs obtained for initial and final (recovered) states after a deformation cycle up to $\varepsilon_{x}=25 \%$ at $400 \mathrm{~K}$. As we can see all initial dPDFs match perfectly as expected in the initial isotropic system. After the shearing cycle, the total PDF (not shown) and the directional ([100], [010] and [001])-dPDFs show small differences

$\Delta g(r)=g(r)_{\text {final }}-g(r)_{\text {initial }}$ between initial and recovered states as shown in the inset plot. The anisotropy, however, is almost undetectable; differences in [100] and [010]dPDFs are exactly the same. Only a small increase in intensity of the $\Delta g(r)$ can be observed in the [001]-dPDF, which corresponds to the PDF of the atoms inside the (001) planes in which all the displacements affine to the applied deformation process take place.

Changes appear when the dPDF are computed in coordinate axes rotated around the [001] direction. Figure 2 shows the computed dPDFs on a $45^{\circ}$ rotated coordinate system, where the maximum differences are found. In this case, the dPDFs are computed on the (110) and $(1 \overline{1} 0)$ planes. The lower dPDFs correspond to the isotropic initial state, while the upper are the final $g(r)$ functions after the deformation cycle. A careful look allows one to distinguish that the three functions are slightly decoupled, indicating the presence of 
remnant anisotropy. This is clearly observed in the inset plot of Fig. 2; the $\Delta g(r)$ functions show higher intensity that the ones corresponding to the (100), (010) and (001) planes and, more interestingly, clear differences in the position of positive and negative maxima can be seen between them.

In order to rationalize the changes in the glass structure induced by the deformation we examined the position of the maxima and the widths of the first three peaks of the PDFs. Figure 3-(a-c) show the peak positions of the [100], [010] and [001]-dPDFs. The right axes indicate the relative changes referred to the $100 \%$ value, given by the average peak position of the three dPDFs in the initial isotropic state. The lack of detectable anisotropy when examining these planes, already stated above, is clearly shown here. The first peak position remains constant after a $10 \%$ deformation cycle, however after $25 \%$ and $40 \%$ cycles it shifts towards shorter $r_{1}$ values. In contrast, the second peak shows a very slight, although perceptible, positive shift for cycles of $25 \%$ and $40 \%$. The position of the third peak moves rather randomly, with an already visible uncertainty in the initial isotropic state. This is due to the less pronounced character of the peak, which results in more uncertainty during the determination of its position by the fitting procedure. Although not at all clear, a slight increase of $r_{3}$ can be observed. The opposite behavior of the first and second atomic shells has been observed experimentally during the expansion of $\mathrm{MG}$ structures, the increase of overall volume per atom is found concurrent with a counterintuitive shrinkage of the position of the first $\mathrm{g}(\mathrm{r})$ maximum, $r_{1}$, and an expansion of the other shells $r_{2}, r_{3}$ and $r_{4}$ [25]. Here, this suggests that the deformation process introduce some amount of excess free volume in the glass structure, as it is confirmed by the expansion of the simulation box during the shearing cycles performed at NPT conditions. At $400 \mathrm{~K}$ the volume per atom changes from 17.430 to $17.431,17.507$ and $17.529 \AA^{3}$ after the $10 \%, 25 \%$ and $40 \%$ deformation cycles, respectively.

To further quantitatively describe the changes on the $g(r)$ functions, the width of each peak, $\Gamma_{i}$, was evaluated by the gap between the left and right intercepts of $g(r)$ and $f(r)=1$ (also shown in Fig. 2). Figure 3-(d-f) shows the clear tendencies to widen and to narrow for the first and second shells respectively. While the widening of the first peak, together with the tendencies of $r_{1}$ and $r_{2}$ discussed before, can be explained by the deformation cycles introducing disorder and expanding the structure, the apparently increase of order at the 
second shell deduced from the decrease of $\Gamma_{2}$ was not at all expected. Analysis of the partial $\mathrm{Cu}-\mathrm{Cu}, \mathrm{Zr}-\mathrm{Zr}, \mathrm{Al}-\mathrm{Al}, \mathrm{Cu}-\mathrm{Zr}, \mathrm{Cu}-\mathrm{Al}$ and $\mathrm{Zr}-\mathrm{Al}$ may shed light on the origin of this overall effect which is related to the differences between the various element environments when adapting to the deformation process. At this moment, however, the reason behind this narrowing of the second shell remains unclear to us. As in the case of $r_{3}$ the calculated values of $\Gamma_{3}$ show a large variability and do not show a clear tendency.

The picture is completely changed when analyzing the dPDFs of the rotated planes. Figure 4-(a-c) and 4-(d-f) show, respectively, the $r_{i}$ and $\Gamma_{i}$ values obtained for the planes (110) and $(1 \overline{1} 0)$, in the same system $(400 \mathrm{~K})$. As before, the effect of deformation in the structure is negligible for the $10 \%$ cycles. For larger deformations, however, the effect of the shearing is clear on the structure. The shift of $r_{1}$ and $\Gamma_{1}$ indicates a clear expansion and increase of disorder of the first atomic shell in the planes (110). These are the planes elongated during the shearing process. On the other hand, the dPDF of the planes (110) shows the clear effect of the applied contraction. The [001]-dPDF shows the average effect on the $x y$-planes; it is interesting to note that while the overall tendency of $r_{1}$ is dominated by the shrinkage of the planes $(1 \overline{1} 0)$, the overall $\Gamma_{1}$ is dominated by the widening tendency of the [110]-dPDF. The changes in the second peak position still clearly show the remnant effects of the elongation and contraction of the box diagonals during the shearing cycles. In this case, however, the overall tendency is dominated by the expansion of $r_{2}$, in agreement with the increase of the average volume per atom after the cycles. Interestingly, the narrowing of $\Gamma_{2}$ is evident in all the families of planes, as it was in Fig. 3-e, indicating the narrowing or ordering of the second shell is an overall behavior related to the adaptation of $\mathrm{Cu}_{46} \mathrm{Zr}_{46} \mathrm{Al}_{8}$ to the deformation process. If this structural change is particular of this composition or is a general behavior in disordered systems is something worth to be elucidated in future work. The third peak position $r_{3}$ shows a similar, but smaller, tendency to the one observed for $r_{2}$, while not noticeable changes in the width $\Gamma_{3}$ can be detected by the current analysis.

The analysis of simulations in the whole range of temperatures show the same qualitative signatures of the induced anisotropy. However, the intensity of these changes is temperature dependent. Fig. 4-g shows the behavior of $\Delta r_{1}=r_{1,[110]}-r_{1,[1 \overline{1} 0]}$ and $\Delta \Gamma_{1}=\Gamma_{1,[110]}-\Gamma_{1,[1 \overline{1} 0]}$, which account for the difference between the positions and widths 
of the peaks of the dPDF in the planes (110) and (11̄0) after a deformation cycle up to $25 \%$. As it can be seen, both the values of $\Delta r_{1}$ and $\Delta \Gamma_{1}$ show an overall decrease as the material's temperature increases. This behavior is expected as the increase of atomic mobility facilitates the equilibration of the deformation signatures. However, the fast shearing rate of the MD simulations makes that anisotropy is not erased even above the glass transition (at $736 \mathrm{~K}$ in this system), thus indicating that the time scale of the experiments is always smaller than the relaxation time of the system. An anomaly in the overall behavior is observed near the glass transition, where $\Delta r_{1}$ increases. Currently we do not know the origin of this slight increase of induced anisotropy at these temperatures.

We will discuss here the results of our simulations in comparison with experimental diffraction studies covering different deformation regimes (elastic, plastic-homogenous, plastic-inhomogeneous) and diverse types of loading configurations (uniaxial, bending, rolling, etc.). Although our simulations are performed in a deformation mode different from these experimental configurations, they can approximate the structural changes expected in the main directions (along and across) relative to the shearing planes appearing in each case. Firstly, however, we will discuss the effect of just thermal expansion. In such case metallic glasses are characterized by a counterintuitive decrease of $r_{1}$ due to the skewness of the first peak generated by the anharmonicity of the interatomic potential [17]. This behavior is not only found in metallic glasses, but also in simple mono-component liquid metals. Therefore, the reduction of the absolute value of $\Delta r_{1}$ with increasing temperature (Figure 4) may come partly from this effect, as both $r_{1,[110]}$ and $r_{1,[1 \overline{1} 0]}$ decrease due to thermal expansion. Conversely, the decrease of the anisotropic differences observed for $r_{i}(i$ $>1$ ) and for all $\Gamma_{i}$ (including $\Gamma_{1}$ ) as temperature increases can only be attributed to the higher ability of the system to relax. It is interesting to note here that in terms of the overall $r_{1}, \Gamma_{1}$ and volume behaviors, the simulated shearing cycles produce a similar effect as to the isotropic thermal expansion. However, the anisotropy revealed by the directional PDFs implies that the microscopic origins of these changes are different.

The elastic deformation regime has been investigated by in situ diffraction experiments [13][26][27]. The anisotropic changes of the first diffraction peak (in the reciprocal space) or of the $r_{i}(i>2)$ values (in the real space) follow the expected elastic strains in agreement with the macroscopic Young's modulus and Poisson's ratio. Instead, 
the changes in the first and second peaks of the $g(r)$ can only be explained by taking into account non-affine SRO rearrangements dependent on the particular elemental composition of the alloy [28]. The contribution of these anelastic or non-affine rearrangements was estimated to be almost $20-25 \%$ of the total apparent elastic strain [29]. In the case of homogeneous deformation or creep under static loads similar anisotropic SRO effects, basically perceived in the first and second PDF peaks, are also found. The remaining anisotropy after creep seems to be originated by similar anisotropic SRO changes as the ones generating the non-affine contribution in the elastic regime [30].

In the inhomogeneous plastic regime the activation of shear bands generates local regions subjected to high degrees of shearing. Heavily deformed samples, by rolling or high-pressure torsion, show a small change in $r_{1}$ and an increase of $\Gamma_{1}$ of the overall $g(r)$ [31][32]. Although these effects have been interpreted as not being compatible with an increase of free volume, we show in this work that an increase of the overall volume after the shearing cycle is in fact compatible with a constant or even smaller $r_{1}$ and a larger $\Gamma_{1}$. The difference of structure between inside and outside shear bands was studied by nanobeam electron diffraction in ref. [33]. They found very small differences in the overall $g(r)$; the width and position of the first peak did not change appreciably while the only observable difference was a slight decrease of the intensity, attributed to a reduction of the SRO. However, as we shown in this work, although the overall $g(r)$ may seem little affected by shearing the system contains significant anisotropic structural differences between the principal directions in agreement with some recent experimental studies [34].

Summarizing, the overall effect found in our simulations is the increase of the volume per atom, the shrinkage of $r_{1}$ and the expansion of the other $g(r)$ peaks, in agreement with the observed structural changes connected to expansion and rejuvenation of metallic glasses [15][25][35]. In addition to the overall effects, the analysis method used in this work reveals very clearly that anisotropy is introduced parallel and perpendicular to the elongation [110] direction. We will note here that, during the deformation process, we observed some localization of deformation in bands. This is one of the characteristics that governs the plastic deformation of MGs as it was shown by Spaepen [36] in his experimental studies. Figure 5 shows the atomic displacements recorded during a deformation cycle. There is an evident localization of the displacements in 'soft' zones. 
This is expected in amorphous structures which are intrinsically characterized by heterogeneity at the nm-scale. The displacement of the atoms localizes in bands basically parallel to the $x z$-planes, precursors of the macroscopic shear banding that will occur parallel to the shear direction. However, the structural modifications seen in the dPDFs show that the anisotropic changes of the atomic structure are along and across the [110] direction and not within the shearing $z x$ planes. This is important to bear in mind when searching anisotropic signatures of plastic deformation after creep or plastic deformation experiments of diverse geometry. The observed remnant anisotropy decreases with temperature, as expected due to enhanced atomic mobility, but the qualitative basic signatures shown by the dPDFs do not change. A future detailed study of the change in relaxation time as function of temperature and how the relation between relaxation time (temperature) and shear rate affect the induction or erosion of remnant anisotropy should allow us to better rationalize the effect of temperature.

\section{Conclusions}

Deformation in a prototypical metallic glass, $\mathrm{Zr}_{46} \mathrm{Cu}_{46} \mathrm{Al}_{8}$, was simulated in order to reveal the remnant anisotropy. Deformation cycles up to a $10 \%, 25 \%$ and $40 \%$, followed by a recovery of the original shape were performed. Alterations in the short and medium range atomic order were exposed by the differential pair distribution functions computed in different orientations. Anisotropy at low (10\%) deformations is only observed in the moderate increase of the position of the second peak of $g(r)$, witness of the generation of free volume in the glass along the shear cycle. Larger deformation cycles, however, induce noticeable changes in the short and medium range orders and introduce significant anisotropy along the [110] and [1피 directions. The above features were observed in all the tested temperatures, although they become progressively reduced as temperature increases. Further work will be devoted to compare to other glass compositions and to check if the observed trends are composition-dependent or are general features of metallic glasses. 


\section{Acknowledgments}

Work funded by MINECO (grant FIS2014-54734-P), Generalitat de Catalunya (grant 2014SGR00581) and Barcelona Supercomputing Center and Cenits-Computaex (grants QCM-2015-1-0011, QCM-2015-2-0017 and QCM-2015-3-0038). J. E. Velasco acknowledges financial support by CONACYT. 


\section{References}

[1] Y. Suzuki, J. Haimovich, T. Egami, Bond-orientational anisotropy in metallic glasses observed by x-ray diffraction, Phys. Rev. B. 35 (1987) 2162-2168. doi:10.1103/PhysRevB.35.2162.

[2] Y. Sun, A. Concustell, A.L. Greer, Thermomechanical processing of metallic glasses: extending the range of the glassy state, Nat. Rev. Mater. 1 (2016) 16039. doi:10.1038/natrevmats.2016.39.

[3] A.L. Greer, Metallic glasses on the threshold, Mater. Today. 12 (2009) 14-22. doi:10.1016/S1369-7021(09)70037-9.

[4] W.H. Wang, Bulk Metallic Glasses with Functional Physical Properties, Adv. Mater. 21 (2009) 4524-4544. doi:10.1002/adma.200901053.

[5] M.D. Demetriou, M.E. Launey, G. Garrett, J.P. Schramm, D.C. Hofmann, W.L. Johnson, R.O. Ritchie, A damage-tolerant glass, Nat Mater. 10 (2011) 123-128. http://dx.doi.org/10.1038/nmat2930.

[6] J. Plummer, Is metallic glass poised to come of age?, Nat. Mater. 14 (2015) 553555. doi:10.1038/nmat4297.

[7] G. Kumar, A. Desai, J. Schroers, Bulk Metallic Glass: The Smaller the Better, Adv. Mater. 23 (2011) 461-476. doi:10.1002/adma.201002148.

[8] A. Concustell, S. Godard-Desmarest, M.A. Carpenter, N. Nishiyama, A.L. Greer, Induced elastic anisotropy in a bulk metallic glass, Scr. Mater. 64 (2011) 1091-1094. doi:10.1016/j.scriptamat.2011.02.028.

[9] Y.H. Sun, A. Concustell, M.A. Carpenter, J.C. Qiao, A.W. Rayment, A.L. Greer, Flow-induced elastic anisotropy of metallic glasses, Acta Mater. 112 (2016) 132140. doi:10.1016/j.actamat.2016.04.022.

[10] E. Pineda, P. Bruna, B. Ruta, M. Gonzalez-Silveira, D. Crespo, Relaxation of rapidly quenched metallic glasses: Effect of the relaxation state on the slow low temperature dynamics, Acta Mater. 61 (2013) 3002-3011. doi:10.1016/j.actamat.2013.01.060.

[11] G. Kumar, P. Neibecker, Y.H. Liu, J. Schroers, Critical fictive temperature for plasticity in metallic glasses (vol 4, pg 1536, 2013), Nat. Commun. 4 (2013) 1536. 
doi:10.1038/ncomms3357.

[12] W. Dmowski, T. Egami, Structural Anisotropy in Metallic Glasses Induced by Mechanical Deformation, Adv. Eng. Mater. 10 (2008) 1003-1007. doi:10.1002/adem.200800121.

[13] T.C. Hufnagel, R.T. Ott, J. Almer, Structural aspects of elastic deformation of a metallic glass, Phys. Rev. B. 73 (2006) 64204. doi:10.1103/PhysRevB.73.064204.

[14] Y. Tong, W. Dmowski, Y. Yokoyama, G. Wang, P.K. Liaw, T. Egami, Recovering compressive plasticity of bulk metallic glasses by high-temperature creep, Scr. Mater. 69 (2013) 570-573. doi:10.1016/j.scriptamat.2013.06.020.

[15] H. Shakur Shahabi, S. Scudino, I. Kaban, M. Stoica, U. Rütt, U. Kühn, J. Eckert, Structural aspects of elasto-plastic deformation of a Zr-based bulk metallic glass under uniaxial compression, Acta Mater. $95 \quad$ (2015) 30-36. doi:10.1016/j.actamat.2015.05.011.

[16] B.S. Shang, M.Z. Li, Y.G. Yao, Y.J. Lu, W.H. Wang, Evolution of atomic rearrangements in deformation in metallic glasses, Phys. Rev. E. 90 (2014) 42303. doi:10.1103/PhysRevE.90.042303.

[17] J. Ding, E. Ma, Computational modeling sheds light on structural evolution in metallic glasses and supercooled liquids, Npj Comput. Mater. 3 (2017) 9. doi:10.1038/s41524-017-0007-1.

[18] H.W. Sheng, W.K. Luo, F.M. Alamgir, J.M. Bai, E. Ma, Atomic packing and shortto-medium-range order in metallic glasses, Nature. 439 (2006) 419-425. doi:10.1038/nature04421.

[19] J.E. Velasco, A. Concustell, E. Pineda, D. Crespo, Plastic deformation induced anisotropy in metallic glasses: A molecular dynamics study, J. Alloys Compd. 707 (2017) 102-107. doi:10.1016/j.jallcom.2016.12.233.

[20] S. Plimpton, Fast Parallel Algorithms for Short-Range Molecular Dynamics, J. Comput. Phys. 117 (1995) 1-19. doi:10.1006/jcph.1995.1039.

[21] Y.Q. Cheng, E. Ma, H.W. Sheng, Atomic Level Structure in Multicomponent Bulk Metallic Glass, Phys. Rev. Lett. $102 \quad$ (2009) 245501. 
doi:10.1103/PhysRevLett.102.245501.

[22] S. Pauly, J. Das, N. Mattern, D.H. Kim, J. Eckert, Phase formation and thermal stability in $\mathrm{Cu}-\mathrm{Zr}-\mathrm{Ti}(\mathrm{Al})$ metallic glasses, Intermetallics. 17 (2009) 453-462. doi:10.1016/j.intermet.2008.12.003.

[23] P. Guan, M. Chen, T. Egami, Stress-Temperature Scaling for Steady-State Flow in Metallic Glasses, Phys. Rev. Lett. $104 \quad$ (2010) 205701. doi:10.1103/PhysRevLett.104.205701.

[24] Y.Q. Cheng, E. Ma, Atomic-level structure and structure-property relationship in metallic glasses, Prog. Mater. Sci. $56 \quad$ (2011) 379-473. doi:10.1016/j.pmatsci.2010.12.002.

[25] X. Tong, G. Wang, Z.H. Stachurski, J. Bednarčík, N. Mattern, Q.J. Zhai, J. Eckert, Structural evolution and strength change of a metallic glass at different temperatures, Sci. Rep. 6 (2016) 30876. doi:doi:10.1038/srep30876.

[26] H.F. Poulsen, J. a. Wert, J. Neuefeind, V. Honkimäki, M. Daymond, Measuring strain distributions in amorphous materials, Nat. Mater. 4 (2005) 33-36. doi:10.1038/nmat1266.

[27] Y.J. Huang, J.C. Khong, T. Connolley, J. Mi, In situ study of the evolution of atomic strain of bulk metallic glass and its effects on shear band formation, Scr. Mater. 69 (2013) 207-210. doi:10.1016/j.scriptamat.2013.03.016.

[28] N. Mattern, J. Bednarčik, S. Pauly, G. Wang, J. Das, J. Eckert, Structural evolution of $\mathrm{Cu}-\mathrm{Zr}$ metallic glasses under tension, Acta Mater. 57 (2009) 4133-4139. doi:10.1016/j.actamat.2009.05.011.

[29] W. Dmowski, T. Iwashita, C.P. Chuang, J. Almer, T. Egami, Elastic heterogeneity in metallic glasses, Phys. Rev. Lett. $105 \quad$ (2010) 2-5. doi:10.1103/PhysRevLett.105.205502.

[30] T. Egami, Y. Tong, W. Dmowski, Deformation in Metallic Glasses Studied by Synchrotron X-Ray Diffraction, Metals (Basel). 6 (2016) 22. doi:10.3390/met6010022.

[31] W. Dmowski, Y. Yokoyama, A. Chuang, Y. Ren, M. Umemoto, K. Tsuchiya, A. 
Inoue, T. Egami, Structural rejuvenation in a bulk metallic glass induced by severe plastic deformation, Acta Mater. $58 \quad$ (2010) 429-438. doi:10.1016/j.actamat.2009.09.021.

[32] R.V. Sundeev, A.M. Glezer, A.P. Menushenkov, A.V. Shalimova, O.V. Chernysheva, N.V. Umnova, Effect of high pressure torsion at different temperatures on the local atomic structure of amorphous Fe-Ni-B alloys, Mater. Des. 135 (2017) 77-83. doi:10.1016/j.matdes.2017.08.062.

[33] Y.M. Chen, T. Ohkubo, T. Mukai, K. Hono, Structure of shear bands in Pd40Ni40P20 bulk metallic glass, J. Mater. Res. 24 (2009) 1-9. doi:10.1557/jmr.2009.0001.

[34] S. Scudino, H.S. Shahabi, M. Stoica, I. Kaban, B. Escher, U. Kühn, G.B.M. Vaughan, J. Eckert, Structural features of plastic deformation in bulk metallic glasses, Appl. Phys. Letts. 106 (2015) 31903.

[35] X.L. Bian, G. Wang, J. Yi, Y.D. Jia, J. Bednarčík, Q.J. Zhai, I. Kaban, B. Sarac, M. Mühlbacher, F. Spieckermann, J. Keckes, J. Eckert, Atomic origin for rejuvenation of a Zr-based metallic glass at cryogenic temperature, J. Alloys Compd. 718 (2017) 254-259. doi:10.1016/j.jallcom.2017.05.124.

[36] F. Spaepen, Metallic glasses: Must shear bands be hot?, Nat. Mater. 5 (2006) 7-8. doi:10.1038/nmat1552. 
Figure Captions

Figure 1. Top) Process of shear deformation up to $25 \%$ at $400 \mathrm{~K}$. Left: initial state after annealing. Right: sheared box. Bottom) Directional [100], [010] and [001]-PDFs before and after the shear deformation cycle at $400 \mathrm{~K}$. In order to help visual comparison, the PDFs are displaced vertically; the initial states below and the final states above. The inset shows the $\Delta \mathrm{g}(\mathrm{r})$ showing differences of the atomic distributions between initial and final states.

Figure 2. Directional [110], [11̄0] and [001]-PDFs before and after the shear deformation cycle at 400 K. Same captions than in Fig. 1.

Figure 3. Positions of the maxima (a-c) and widths (d-f) of the [100], [010] and [001]-dPDF peaks after complete shear deformation cycles with different values of maximum shear (10, 25 and $40 \%$ ). The value of $0 \%$ shear correspond to the non-deformed initial state. From top to bottom, first second and third peak.

Figure 4. Positions of the maxima (a-c) and widths (d-f) of the [110], [110] and [001]-PDF peaks after complete shear deformation cycles with different values of maximum shear (10, 25 and $40 \%$ ). The value of $0 \%$ shear correspond to the non-deformed initial state. From top to bottom, first second and third peak. g) Evolution with temperature of the differences between [110] and [1 $\overline{1} 0]$-dPDFs in position of the maximum, $\Delta r_{1}$, and width, $\Delta \Gamma_{1}$, of the first peak.

Figure 5. Non-affine atomic displacements from the original initial state during a shear deformation cycle at $400 \mathrm{~K}$. Lighter colored atoms correspond to larger displacements. 
Highlights

- Molecular dynamics simulation of shear deformation in $\mathrm{CuZrAl}$ glass former

- Analysis of shear deformation cycle effects on the atomic structure of the glass

- Remnant anisotropy revealed by directional pair distribution functions

- Anisotropy perceptible along and across $45^{\circ}$ respect to the shear direction 
Figure 2

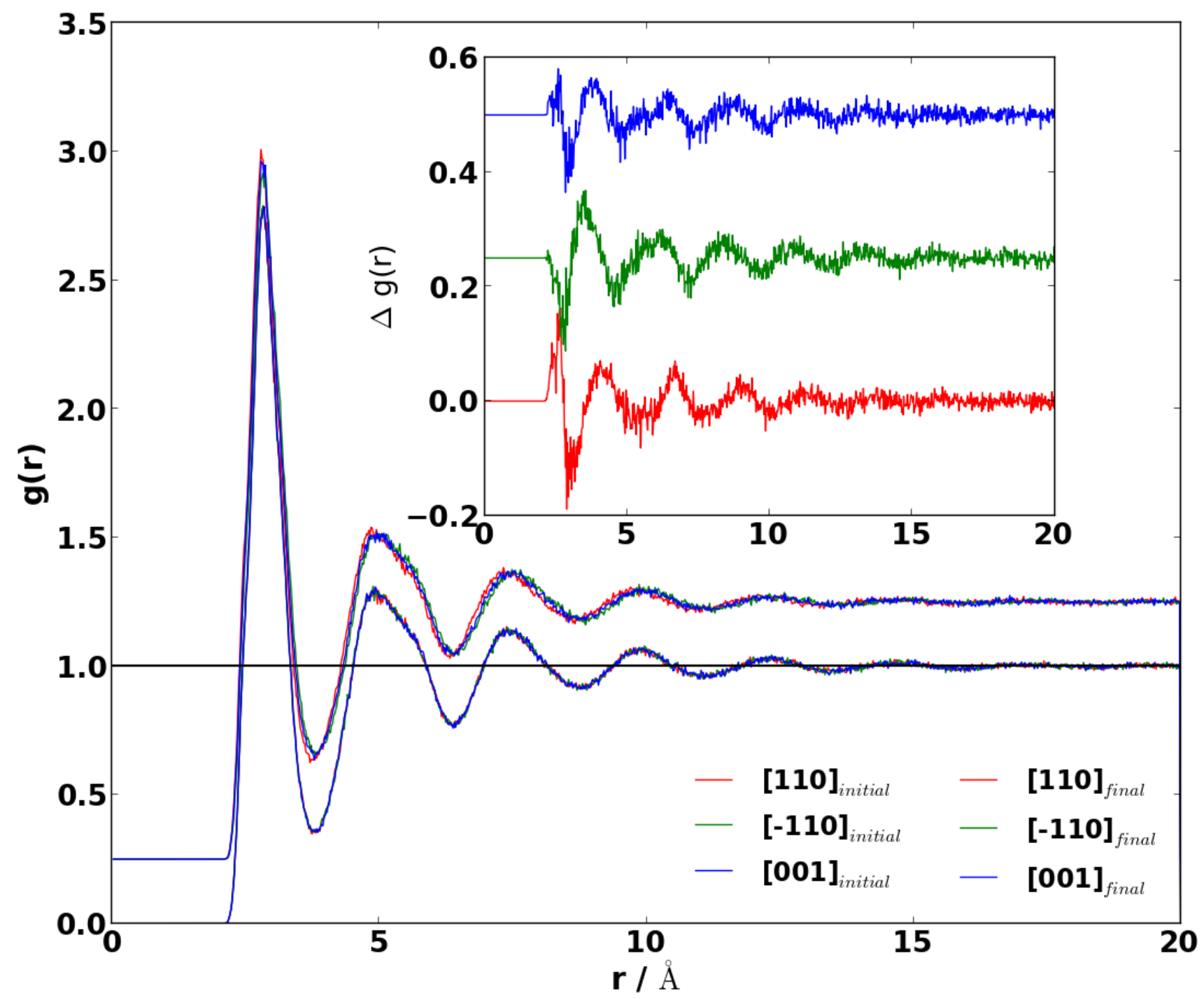

Figure 2 

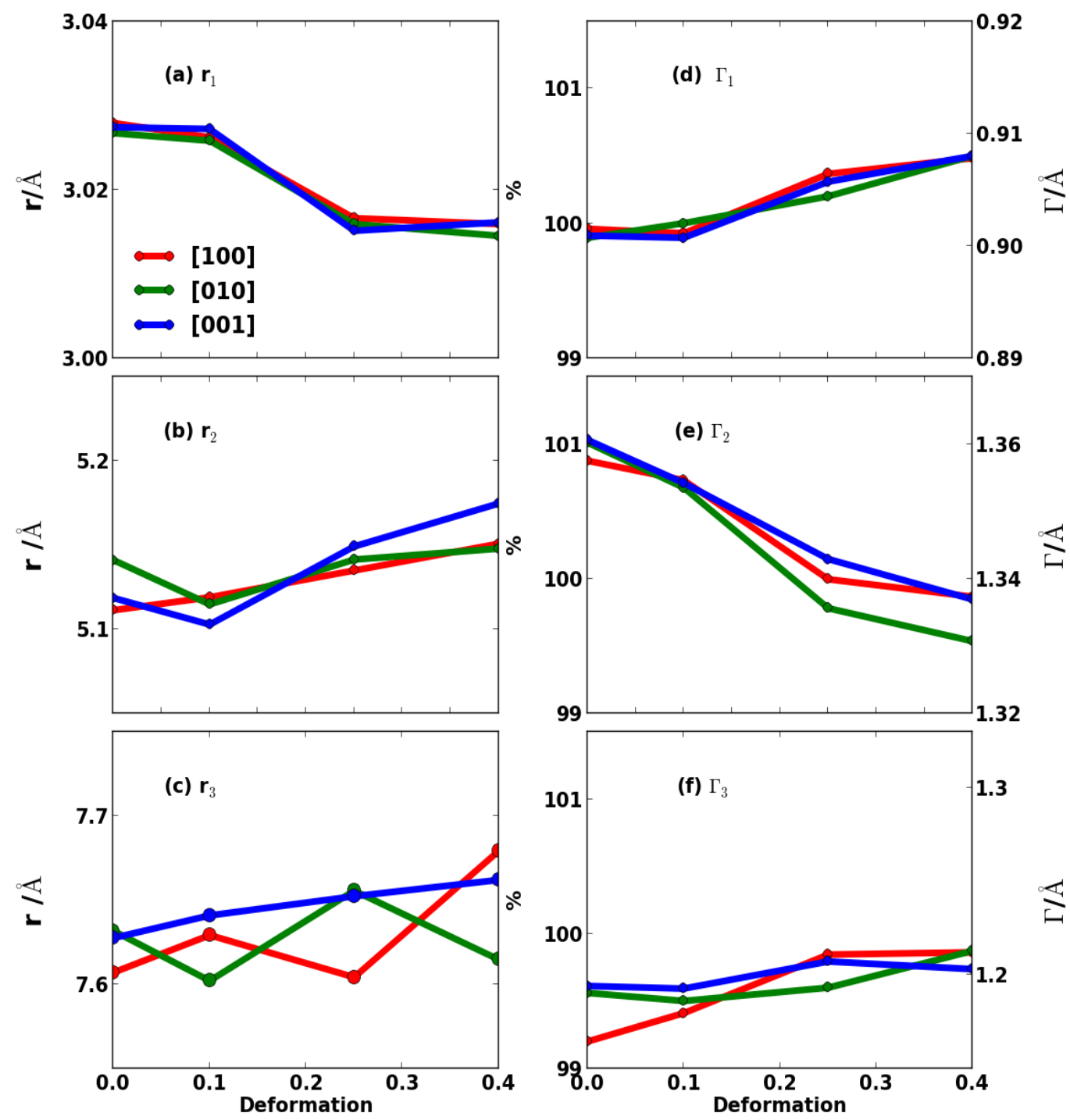

Figure 3 

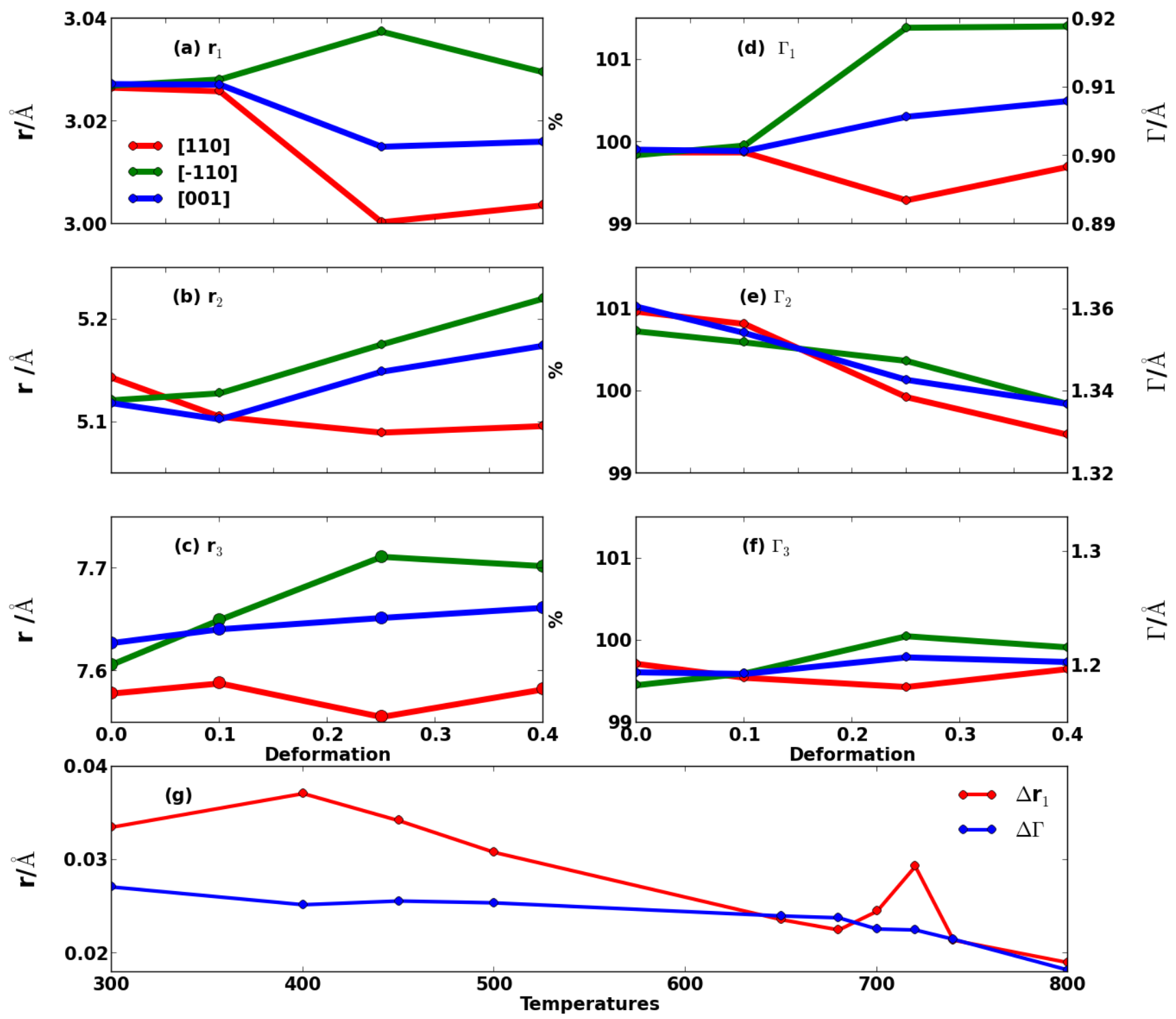

Figure 4 
